\title{
Multiple Reflections and Fresnel Absorption of Gaussian Laser Beam in an Actual 3D Keyhole during Deep-Penetration Laser Welding
}

\author{
Xiangzhong Jin, Yuanyong Cheng, Licheng Zeng, Yufeng Zou, and Honggui Zhang \\ State Key Laboratory of Advanced Design and Manufacturing for Vehicle Body, Hunan University, Hunan, Changsha 410082, China \\ Correspondence should be addressed to Xiangzhong Jin, jin9000xz@hotmail.com
}

Received 13 February 2012; Accepted 26 March 2012

Academic Editor: Hongming Zhao

Copyright (C) 2012 Xiangzhong Jin et al. This is an open access article distributed under the Creative Commons Attribution License, which permits unrestricted use, distribution, and reproduction in any medium, provided the original work is properly cited.

In deep penetration laser welding, a keyhole is formed in the material. Based on an experimentally obtained bending keyhole from low- and medium-speed laser penetration welding of glass, the keyhole profiles in both the symmetric plane are determined by polynomial fitting. Then, a 3D bending keyhole is reconstructed under the assumption of circular cross-section of the keyhole at each keyhole depth. In this paper, the behavior of focused Gaussian laser beam in the keyhole is analyzed by tracing a ray of light using Gaussian optics theory, the Fresnel absorption and multiple reflections in the keyhole are systematically studied, and the laser intensities absorbed on the keyhole walls are calculated. Finally, the formation mechanism of the keyhole is deduced.

\section{Introduction}

When laser beam with high laser intensity irradiates work piece, the material is vaporized, recoil pressure is produced, and a keyhole is formed in the material. Then, laser beam can directly enter the keyhole and propagate in the keyhole by means of multiple reflections on the keyhole wall, and the laser energy is absorbed through the Fresnel absorption. The formation of keyhole enhances the energy-coupling efficiency between laser beam and material.

Concerning the Fresnel absorption and multiple reflections in the keyhole, a lot of work has been done. In 1989, Dowden et al. [1] considered the Fresnel absorption, but the way they used is too simple with a uniform laser line source. In 1990, Beck et al. [2] traced rays in a symmetric keyhole at low speed and established a numerical model of the Fresnel absorption. In 1992 and in 1996, Kern [3] and Mueller [4], simulated the Fresnel absorption and multiple reflections in a predetermined 3D keyhole, respectively. In 1994, Kaplan [5] developed a model to calculate the asymmetric keyhole profile and considered the Fresnel absorption and multiple reflections in the keyhole in a simple way. In 1997, Solana and Negro [6] used an axisymmetrical model to analyze keyhole profiles and laser intensity distributions with keyhole depth for the cases of a Gaussian and a uniform top-hat distribution laser beam and studied the effect of multiple reflections inside the keyhole with a free boundary. In 2000, Fabbro and Chouf [7] studied multiple reflections inside the keyhole by tracing a ray. But in all of the above work, the real keyhole shapes were dealt with some simple ways. For example, the keyhole was assumed to be rotationally symmetric. But it is different from reality. In practical deep penetration laser welding, especially in high-speed penetration laser welding, the keyhole is not rotationally symmetric anymore, but bent and elongated. So their results may have great difference from real cases.

To study the Fresnel absorption and multiple reflections in the real keyhole during laser penetration welding, in 1995 and in 1997, Semak et al. [8] and Katayama et al. [9] observed the formation and collapse times of the keyhole from top view with a high-speed camera in case of laser welding stainless steel and aluminum alloy, respectively. In 1999, Fujinaga et al. [10] observed directly the actual keyhole with an in situ microfocused X-ray transmission imaging system. Because of unsuitable material aluminum alloy used, the keyhole is not clear enough. In 2002, Jin et al. [11] improved the experimental setup of Arata et al. [12]. With this setup and 
specially selected transparent material (GG17 glass, a Chinese brand of glass similar to Pyrex glass), they observed clearly the keyhole during deep penetration laser welding of GG17 glass. On the basis of a so-obtained keyhole picture, the keyhole profile was determined by means of polynomial fitting. Then, using geometrical optics theory, Jin et al. traced a ray of light in the keyhole and calculated the laser intensity absorbed on the keyhole wall in the symmetrical plane including laser beam and welding velocity through the Fresnel absorption. In 2006, 2008, and 2012, under the assumption of circular and elliptical cross-section of the keyhole at each keyhole depth, respectively, Jin et al. [13-15] studied multiple reflections and the Fresnel absorption in the actual bending 3D keyhole during deep-penetration laser welding and computed the laser intensity absorbed on the 3D keyhole wall. The results of Jin et al.'s above work indicated that laser intensity absorbed on the keyhole walls through the Fresnel absorption is unevenly distributed and mainly absorbed on the front keyhole wall. But in all of Jin et al.'s above work, geometrical optics theory is used to trace a ray of light in the keyhole. Actually, laser beam is a Gaussian beam. When a ray of Gaussian beam propagates in the keyhole, its propagation track should be determined according to Gaussian optics theory. In other words, the propagation track of the ray is not a line, but a hyperboloid. Using geometrical optics theory to trace a ray of light in the keyhole is just an approach method. So Jin's results may have some differences from the real case.

In this paper, based on an experimentally obtained bending keyhole by improving Jin's keyhole model [13], Gaussian optics theory, instead of geometrical optics theory, is used to study the Fresnel absorption and multiple reflections in the keyhole. The laser intensity absorbed on the keyhole wall through the Fresnel absorption is calculated.

\section{Multiple Reflections in the 3D Keyhole}

The keyhole picture used in this paper is the same as that in [13]. Followed the same idea as that in [13], the keyhole profile can be determined and a $3 \mathrm{D}$ keyhole can be reconstructed, which are shown in Figures 1(a) and 1(b), respectively. This 3D keyhole is used for later calculation.

According to Guo et al.'s work [16], when the Gaussian beam falls directly on the surface, the reflected ray is still bending, which is shown in Figure 2. The reflected ray is symmetrical to the extension of the incident ray. The incident-optical axis is symmetrical to reflected-optical axis about the normal line to the plane MN-like geometrical optics.

So when a ray of light arrives directly at the point $p$, it is reflected as shown in Figure 3.

The focal plane of the focused Gaussian beam is assumed to be on the surface of the sample. In other words, the waist of the Gaussian beam is located on the surface of the sample.

Following the same way as that in [13], when laser beam irradiates into the $3 \mathrm{D}$ keyhole, picking up any three neighboring nodes $A\left(x_{1}, y_{1}, z_{1}\right), B\left(x_{2}, y_{2}, z_{2}\right)$, and $C\left(x_{3}, y_{3}, z_{3}\right)$ from the keyhole wall, the equation of the small plane consisting of these three points can be derived as follows (as shown in Figure 4).

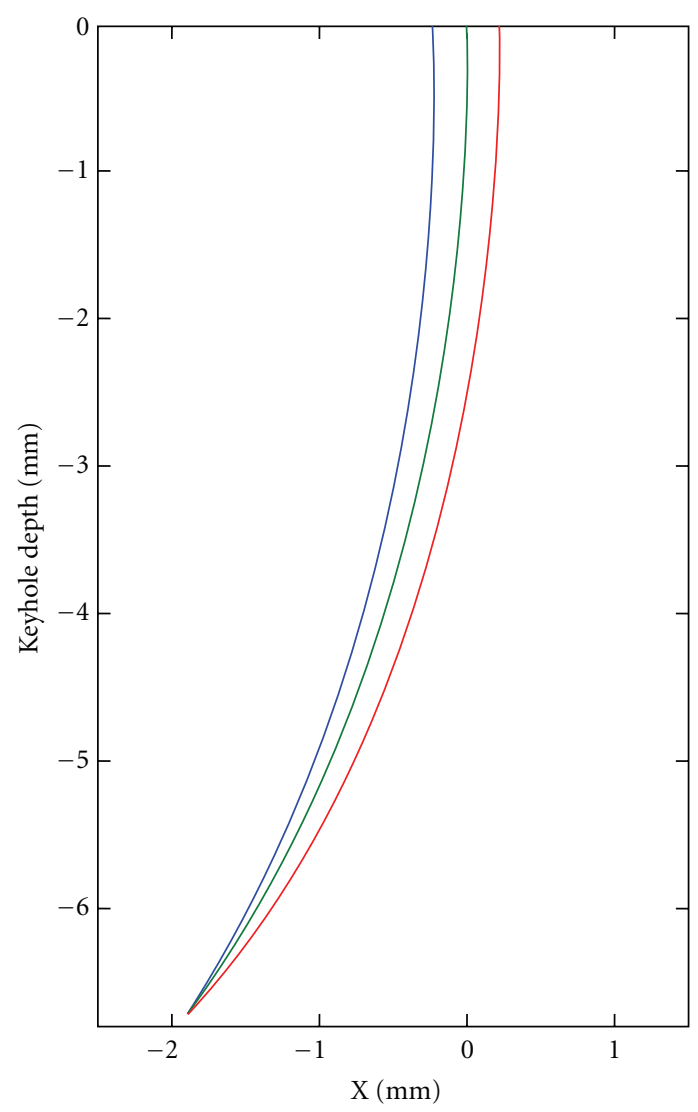

(a)

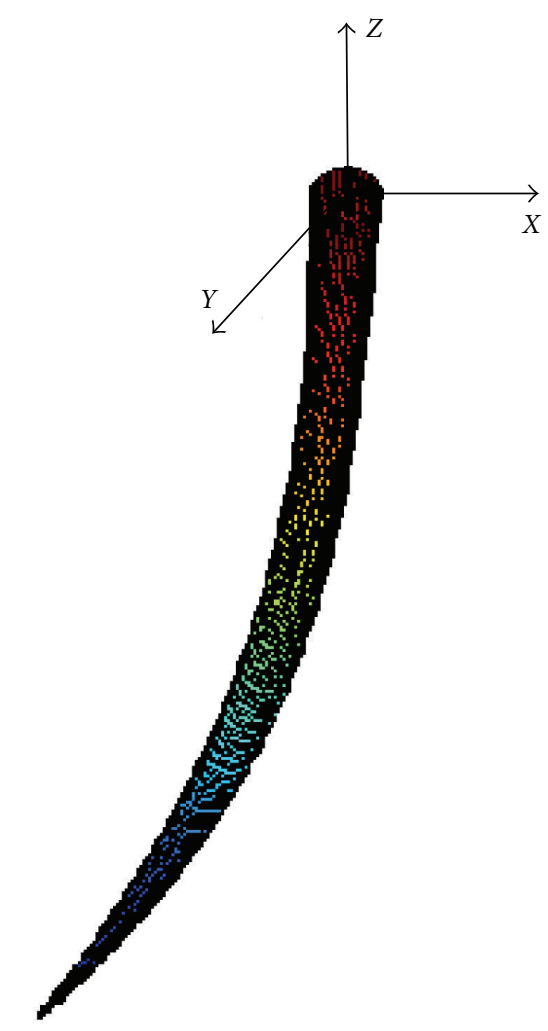

(b)

Figure 1: Diagram of keyhole profile and 3D keyhole. 


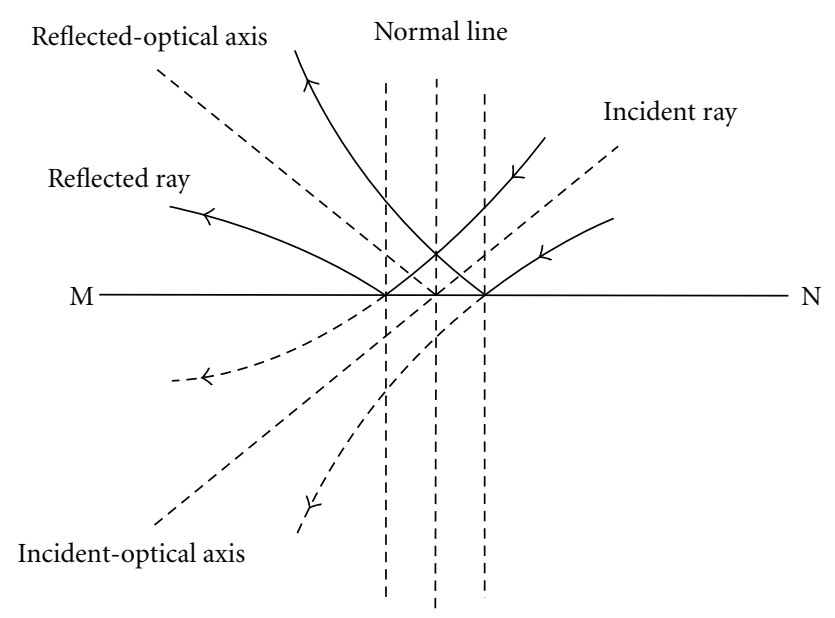

FIgURE 2: A diagram of plane reflection.

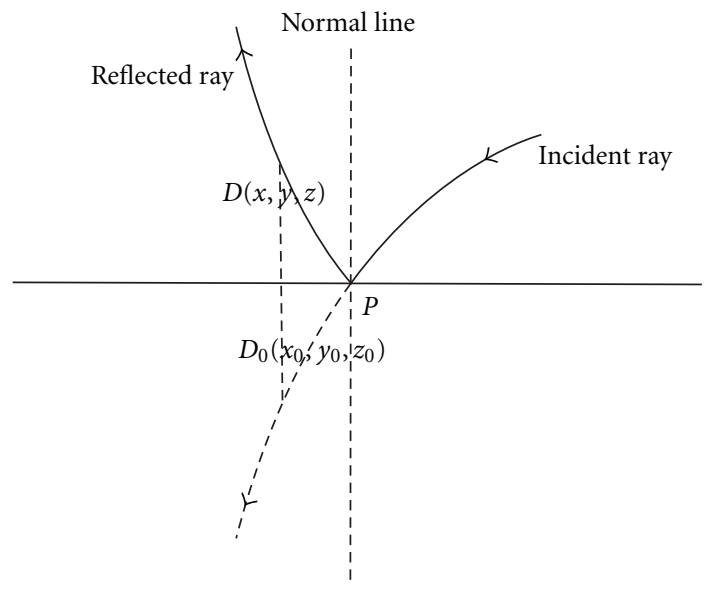

Figure 3: A diagram of the reflected ray.

The normal vector of the plane $A B C$ can be expressed as the cross product of vector $R_{A B}\left(x_{2}-x_{1}, y_{2}-y_{1}, z_{2}-z_{1}\right)$ and vector $R_{B C}\left(x_{3}-x_{2}, y_{3}-y_{2}, z_{3}-z_{2}\right)$ :

$$
\overrightarrow{n_{p}}=\overrightarrow{R_{A B}} \times \overrightarrow{R_{B C}} .
$$

In order to simplify the following calculation, the vector $\overrightarrow{n_{p}}$ can be normalized and represented by a unit vector $\overrightarrow{n_{p}}\left(n_{x}\right.$, $\left.n_{y}, n_{z}\right)$.

Suppose $P$ is a point on the plane $A B C$, whose coordinates $p\left(x_{p}, y_{p}, z_{p}\right)$ are

$$
\begin{aligned}
& x_{p}=\frac{x_{1}+x_{2}+x_{3}}{3}, \\
& y_{p}=\frac{y_{1}+y_{2}+y_{3}}{3}, \\
& z_{p}=\frac{z_{1}+z_{2}+z_{3}}{3} .
\end{aligned}
$$

So the equation of the plane $A B C$ can be expressed as

$$
\left(x-x_{p}\right) n_{x}+\left(y-y_{p}\right) n_{y}+\left(z-z_{p}\right) n_{z}=0 .
$$

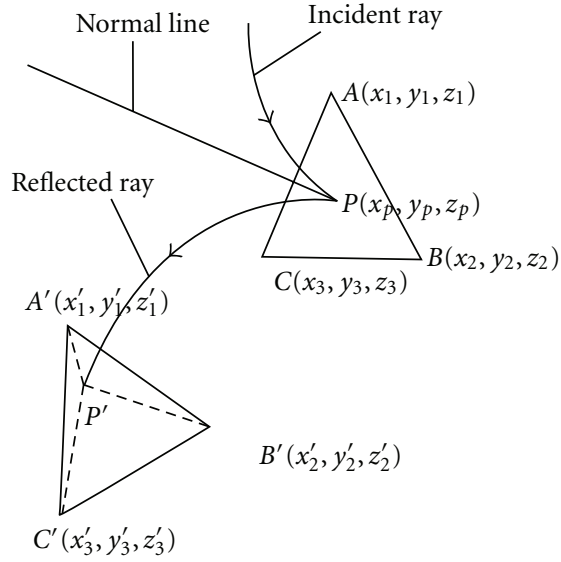

Figure 4: A diagram of multiple reflections.

Following the same procedure, equations of all the planes consisting of any the three neighboring nodes can be obtained.

The distance between point $p$ and the optical axis is

$$
r_{0}=\sqrt{x_{p}^{2}+y_{p}^{2}}
$$

If the ray that arrives at the point $p$ comes from the point $w$ on the waist of Gaussian beam, the distance between the point $w$ and coordinates origin is

$$
r_{1}=\frac{r_{0}}{\sqrt{1+\left(z_{p} / f\right)^{2}}},
$$

where $f$ is focal length of the lens.

The Gaussian beam meets the following hyperboloid equation:

$$
\frac{x^{2}}{a^{2}}+\frac{y^{2}}{a^{2}}-\frac{z^{2}}{b^{2}}=1
$$

For every ray arriving at a point, $a$ and $b$ are constant. For the point $w, a=r_{1}$. And then, after the coordinates of point $p\left(x_{p}, y_{p}, z_{p}\right)$ are substituted into the equation, the constant $b$ can be solved. So the equation of the ray, which passes through $w$ and then arrives at the point $p$, can be obtained. Following the same way, all equations of rays emitted from the Gaussian beam waist can be deduced.

Now, the ray of light that arrives directly at the point $p\left(x_{p}, y_{p}, z_{p}\right)$ is traced to see how it propagates in the $3 \mathrm{D}$ keyhole.

At first, the first incidence of the ray is considered. Following the above way, the equation of the ray that arrives directly at the point $p$ can be obtained, and then $a$ and $b$ can be calculated. Just as showed in Figure 3, the extension of the incident ray passes through point $D_{0}\left(x_{0}, y_{0}, z_{0}\right)$. Correspondingly, there is a point $D(x, y, z) . D_{0}$ and $D$ are symmetrical to the plane $A B C$, and the line passing through $D_{0}$ and $D$ is 
perpendicular to the plane $A B C$. So the following equations can be got:

$$
\begin{gathered}
n_{x}\left(\frac{x+x_{0}}{2}-x_{p}\right)+n_{y}\left(\frac{y+y_{0}}{2}-y_{p}\right)+n_{z}\left(\frac{z+z_{0}}{2}-z_{p}\right)=0, \\
\frac{x-x_{0}}{n_{x}}=\frac{y-y_{0}}{n_{y}}=\frac{z-z_{0}}{n_{z}} .
\end{gathered}
$$

Solving simultaneously (7), the coordinates of point $D_{0}\left(x_{0}, y_{0}, z_{0}\right)$ can be calculated, namely, $x_{0}, y_{0}$, and $z_{0}$ can be expressed by $x, y$, and $z$ which are the coordinates of point $D(x, y, z)$. In addition, the extension of incident ray passes through $D_{0}$, so $x_{0}, y_{0}$, and $z_{0}$ satisfy the equation

$$
\frac{x_{0}^{2}}{a^{2}}+\frac{y_{0}^{2}}{a^{2}}-\frac{z_{0}^{2}}{b^{2}}=1
$$

Definitely, the first incident ray can be expressed with two equations as follows:

$$
\begin{aligned}
& \frac{x_{0}}{a \sqrt{1+\left(z_{0} / b\right)^{2}}}=\cos \phi, \\
& \frac{y_{0}}{a \sqrt{1+\left(z_{0} / b\right)^{2}}}=\sin \phi,
\end{aligned}
$$

where $\phi$ is determined by the first incident point of the incident ray, which is constant. Take the ray arriving at the point $p$ as an example, $\cos \phi=x_{p} / r_{0}$ and $\sin \phi=y_{p} / r_{0}$.

With the following definition, the tangential vector $\vec{n}_{\text {in } 0}\left(n_{0 x}, n_{0 y}, n_{0 z}\right)$ of the incident ray at the point $p$ can be obtained:

$$
\begin{aligned}
& F(x, y, z)=x-a \sqrt{1+\left(\frac{z}{b}\right)^{2}} \cos \varphi, \\
& G(x, y, z)=x-a \sqrt{1+\left(\frac{z}{b}\right)^{2}} \sin \varphi,
\end{aligned}
$$

where $n_{0 x}, n_{0 y}, n_{0 z}$ can be described as follows:

$$
\begin{aligned}
& n_{0 x}=\left|\begin{array}{ll}
F_{y} & F_{z} \\
G_{y} & G_{z}
\end{array}\right|_{p}, \\
& n_{0 y}=\left|\begin{array}{ll}
F_{z} & F_{x} \\
G_{z} & G_{x}
\end{array}\right|_{p}, \\
& n_{0 z}=\left|\begin{array}{ll}
F_{x} & F_{y} \\
G_{x} & G_{y}
\end{array}\right|_{p},
\end{aligned}
$$

where $F_{x}, F_{y}, F_{z}$ and $G_{x}, G_{y}, G_{z}$ are the partial derivative to $x, y, z$ of the two functions $F(x, y, z)$ and $G(x, y, z)$, respectively.

The incident angle $\varphi_{1}$ of the ray equals the angle between the incident ray's tangential line and the normal line of the plane $A B C$, which can be calculated from the dot product of the unit-tangential vector $\vec{n}_{\text {in } 1}\left(n_{1 x}, n_{1 y}, n_{1 z}\right)$ of incident ray and the unit normal vector $\overrightarrow{n_{p}}$ of the plane, that is,

$$
\cos \left(\varphi_{1}\right)=-\vec{n}_{\text {in } 1} \times \vec{n}_{p},
$$

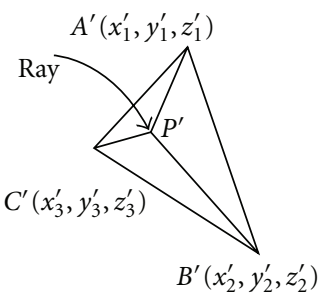

(a)

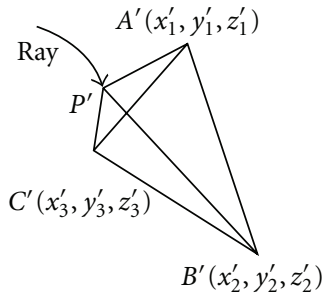

(b)
Figure 5: Diagram of the position determination of the reflected ray.

where "-" represents the opposite direction of the vector $\vec{n}_{\text {in } 1}$.

Then, with (9), when $x_{0}, y_{0}$, and $z_{0}$ are replaced by $x, y$, and $z$, the equation of reflected ray can be calculated.

Now, the point at which the reflected ray falls can be determined.

The second incident ray is the reflected light of the firstincident ray. As is shown in Figure 4, solving simultaneously the equation of second incident ray and the equation of the plane consisting of any three neighboring nodes $A^{\prime}\left(x_{1}^{\prime}\right.$, $\left.y_{1}^{\prime}, z_{1}^{\prime}\right), B^{\prime}\left(x_{2}^{\prime}, y_{2}^{\prime}, z_{2}^{\prime}\right)$ and $C^{\prime}\left(x_{3}^{\prime}, y_{3}^{\prime}, z_{3}^{\prime}\right)$, the coordinates of the intersection points between the second incident ray and all the small planes can be calculated. The intersection point between the second incident ray and the small plane is denoted as $p^{\prime}\left(x_{2 \text { in }}^{\prime}, y_{2 \text { in }}^{\prime}, z_{2 \text { in }}^{\prime}\right)$.

Figure 5 shows the two cases that the second incident ray strikes the region of the small triangle $A^{\prime} B^{\prime} C^{\prime}$. With the known coordinates of the nodes $A^{\prime}\left(x_{1}^{\prime}, y_{1}^{\prime}, z_{1}^{\prime}\right), B^{\prime}\left(x_{2}^{\prime}, y_{2}^{\prime}, z_{2}^{\prime}\right)$, $C^{\prime}\left(x_{3}^{\prime}, y_{3}^{\prime}, z_{3}^{\prime}\right)$, and the intersection point $P^{\prime}\left(x_{2 \text { in }}^{\prime}, y_{2 \text { in }}^{\prime}, z_{2 \text { in }}^{\prime}\right)$, the areas of such triangles as $A^{\prime} B^{\prime} C^{\prime}, A^{\prime} P^{\prime} C^{\prime}, A^{\prime} P^{\prime} B^{\prime}$, and $B^{\prime} P^{\prime} C^{\prime}$ can be calculated. If the second incident point $P^{\prime}$ of the ray is located in the region of triangle $A^{\prime} B^{\prime} C^{\prime}$, the coordinates of point $P^{\prime}\left(x_{2 \text { in }}^{\prime}, y_{2 \text { in }}^{\prime}, z_{2 \text { in }}^{\prime}\right)$ must satisfy the following condition:

$$
S_{A^{\prime} B^{\prime} C^{\prime}}=S_{A^{\prime} P^{\prime} B^{\prime}}+S_{A^{\prime} P^{\prime} C^{\prime}}+S_{B^{\prime} P^{\prime} C^{\prime}} .
$$

Otherwise, if

$$
S_{A^{\prime} B^{\prime} C^{\prime}}<S_{A^{\prime} P^{\prime} B^{\prime}}+S_{A^{\prime} P^{\prime} C^{\prime}}+S_{B^{\prime} P^{\prime} C^{\prime}}
$$

the second incident ray will reach into other parts of the keyhole.

Following the same way above, equation of the $(k-1)$ th incident ray can be expressed with coordinates of the $k$ th incident ray, and equation of the $(k-2)$ th incident ray can be expressed with coordinates of the $(k-1)$ th incident ray. So the equation of the $(k-2)$ th incident ray can be expressed with coordinates of the $k$ th incident ray. Continuingly, the first incident ray can be expressed by coordinates of the $k$ th incident ray. If the coordinates of $k$ th incident point are $\left(x_{k i n}, y_{k i n}, z_{k i n}\right)$, correspondingly there is a point 
$\left(x_{\text {lin }}^{\prime \prime}, y_{\text {lin }}^{\prime \prime}, z_{\text {lin }}^{\prime \prime}\right)$ in the first incident ray, which can be expressed with functions as follows:

$$
\begin{gathered}
x_{\text {lin }}^{\prime \prime}=F X\left(x_{k \text { in }}, y_{k \text { in }}, z_{k \text { in }}\right), \\
y_{\text {lin }}^{\prime \prime}=F Y\left(x_{k \text { in }}, y_{k \text { in }}, z_{k \text { in }}\right), \\
z_{\text {lin }}^{\prime \prime}=F Z\left(x_{k \text { in }}, y_{k \text { in }}, z_{k \text { in }}\right) .
\end{gathered}
$$

So the equation of the $k$ th incident ray can be obtained, then the coordinates of the $k$ th incident point can be determined, and the $k$ th incident angle $\varphi_{k}$ can be calculated.

The Gaussian beam intensity at radius $r$ inside its waist is

$$
I(r)=\frac{2 P}{\pi \omega^{2}} e^{\left(-2 r^{2} / \omega^{2}\right)},
$$

where $P$ is the power of the laser beam, and $\omega$ is the beam radius at waist. For a fixed ray, $r=a$.

The beam intensity at the point of keyhole wall, where the ray reaches for the $i$ th incidence, is

$$
I_{i}=\prod_{j=1}^{i-1} R\left(\phi_{j}\right) \frac{I(r)}{1+\left(z_{1 \text { in }}^{\prime \prime} / b\right)^{2}},
$$

where, with function (17), $z_{\text {lin }}^{\prime \prime}$ is expressed by the coordinates $\left(x_{i \mathrm{in}}, y_{i \mathrm{in}}, z_{\text {iin }}\right)$ of the $i$ th incident point and $R\left(\varphi_{j}\right)$ is the reflectivity of the $j$ th reflection at the keyhole wall.

The absorbed intensity at the point of the keyhole wall from the $i$ th reflection is

$$
I_{i a b}=\left(1-R\left(\phi_{i}\right)\right) I_{i} \cos \left(\phi_{i}\right) .
$$

The total laser intensity absorbed at a point on the keyhole wall from the first $k$ times reflection is

$$
I_{a b}=\sum_{i=1}^{k} I_{i a b}
$$

\section{Results and Discussions}

To calculate the absorbed laser intensity, the reflectivity of the glass at different incident angles must be known first. In the following calculation, the experimental reflectivity of glass GG17 used in [13] is adopted.

The calculation conditions are listed here: laser beam power $P=600 \mathrm{~W}$; the focus of the lens $f=100 \mathrm{~mm}$; Gaussian beam waist radius $w=0.21 \mathrm{~mm}$. The polynomial fitting coefficients of the front, rear keyhole wall, and central line of the keyhole (both $x$ and $z$ is in unit of meter) are as follows:

$$
\begin{array}{cc}
a_{1}=7476.47680, & a_{2}=19.29279, \\
a_{3}=0.09948, & a_{4}=0.00023, \\
b_{1}=2907.51578, & b_{2}=-17.15273, \\
b_{3}=0.00069, & b_{4}=-0.00022 .
\end{array}
$$

The fitting keyhole profile and its central line are shown in Figure 1(a), which is used for further study. In the following calculation, the whole keyhole wall, as shown in

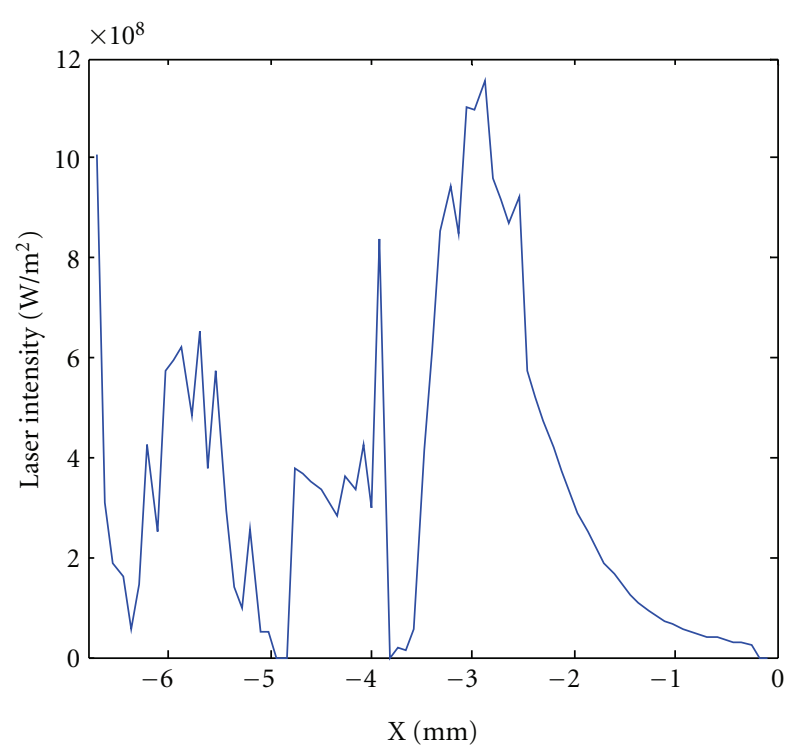

FIgURE 6: The laser intensity absorbed on the front keyhole wall.

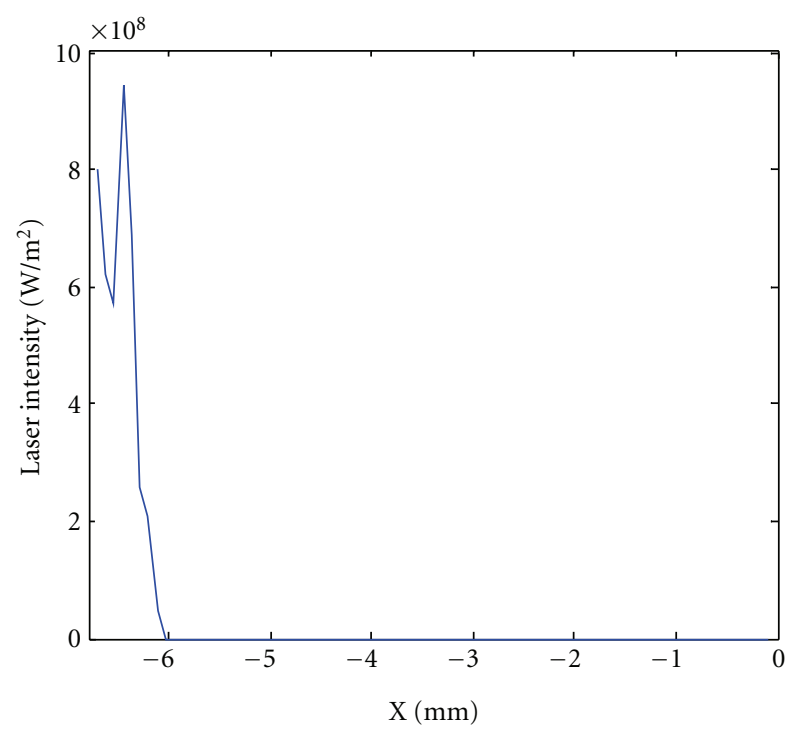

FIGURE 7: The laser intensity absorbed on the rear keyhole wall.

Figure $1(\mathrm{~b})$, is divided into $79 \times 118$ small triangles, where 79 represents the number of layers along the direction of the keyhole depth and 118 represents the number of divisions along the angular direction at each keyhole depth.

Figures 6 and 7 shows that the laser intensity distributes on the front and rear keyhole wall, respectively. It can be seen clearly that far more laser intensity is absorbed by the front wall. The reason is obvious when Figure 8 is referred, which shows the diagram of incident rays on the keyhole profile for the first three times. Clearly, the rays of laser beam can fall on the front wall directly, and only on the bottom of keyhole, they are reflected to the rear wall. Thus, on the keyhole wall from depth about $6 \mathrm{~mm}$ upward, there is almost no laser intensity absorbed on the rear wall. On the contrary, the distribution of laser intensity is extensive on the front 


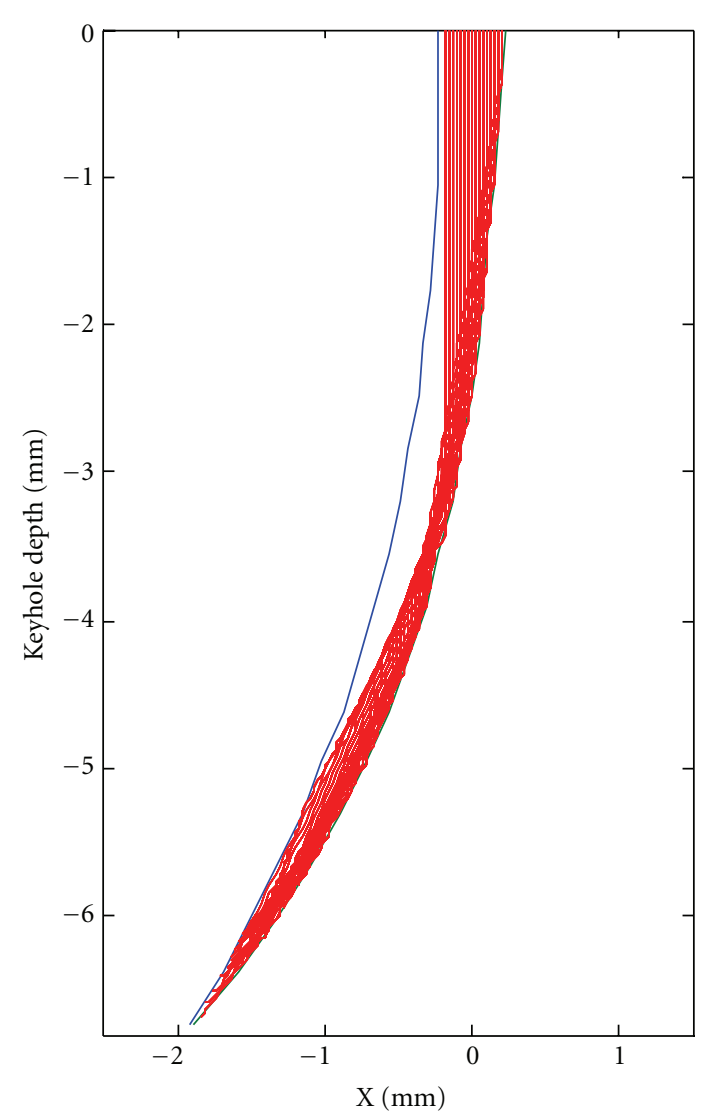

FIGURE 8: Diagram of incident rays on keyhole profile.

keyhole wall. However, the laser intensities absorbed both on the front and rear keyhole walls are very high; the values of them are almost the same. The reason for this phenomenon involves incident angle and multiple reflections. From Figure 9 which shows the diagram of a ray reflected on the keyhole profile, it can be found that when the ray pentrates into the keyhole, after two or three times reflections, it will reach the keyhole bottom and be trapped there so at the bottom of the keyhole, it is reflected more frequently than that on the upper part of the keyhole. In addition, the incline of the keyhole increases as the keyhole depth increases so when a ray is reflected to the bottom of the keyhole, the incident angles decreases and the absorption coefficient increases. Therefore, both the front and rear walls absorb almost the same laser intensity at the keyhole bottom.

As shown in Figure 6, the distribution of the laser intensity absorbed on the front keyhole wall is very uneven. This phenomenon can be easily explained. The first incident rays irradiate on the front keyhole wall from keyhole mouth to keyhole depth about $3.6 \mathrm{~mm}$, and the center of laser beam irradiates near depth $3 \mathrm{~mm}$. Therefore, due to the incline of the keyhole and Gaussian distribution of laser beam, high laser intensity is absorbed from keyhole depth $3.6 \mathrm{~mm}$ upward, with a peak of $11.5 \times 10^{8} \mathrm{~W} / \mathrm{m}^{2}$ occurring at depth near $3 \mathrm{~mm}$. Besides, there is absorption low ebb near depth $3.7 \mathrm{~mm}$. When Figure 8 is magnified from depth about $2.4 \mathrm{~mm}$ to $4.8 \mathrm{~mm}$, which is shown in Figure 10, it is clear

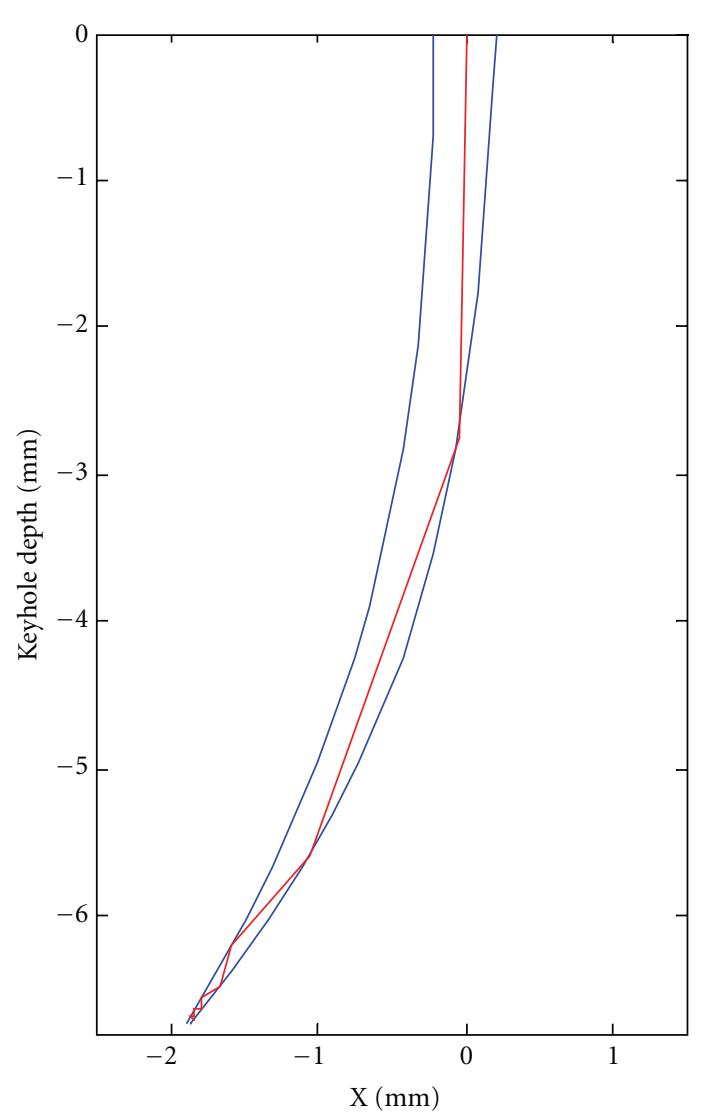

Figure 9: Diagram of a ray reflected on the keyhole profile.

that no first incident rays strike some area near depth $3.7 \mathrm{~mm}$, so the absorption low ebb forms. Additionally, there is another low ebb at depth about $4.9 \mathrm{~mm}$. However, the reason for this low ebb is different from that at depth about $3.7 \mathrm{~mm}$. It can be seen clearly from Figure 11, which is a part of Figure 8. Some rays can be reflected to the area from depth about $4.8 \mathrm{~mm}$ to $5.1 \mathrm{~mm}$. But the reflected rays are very sparse and the incident angles are very big. The phenomenon of incident angle near 90 degrees is known as grazing incidence. Under this circumstance, the laser reflectivity of GG17 glass is approaching $100 \%$, which was shown in Figure 5 of [11]. Due to the above two reasons, little laser intensity can be absorbed by the front keyhole wall near depth $4.9 \mathrm{~mm}$. Therefore, the absorption low ebb near depth 4.9 forms. Moreover, due to multiple reflections, the laser intensity absorbed at the bottom of front keyhole wall can be as much as that on the upper part.

Figure 12 shows the distribution of average laser intensity absorbed along keyhole depth. From this figure, it is easily to explain how laser power is absorbed by the Fresnel absorption along keyhole depth. Laser intensity absorbed on the keyhole wall has such a tendency that, from the top to depth about $5.5 \mathrm{~mm}$ of the keyhole, laser intensity absorbed ascends first, with a peak at $1.5 \times 10^{8} \mathrm{~W} / \mathrm{m}^{2}$ near depth $3 \mathrm{~mm}$ and then descends gradually, which is similar to parabolic distribution. And then it ascends sharply, reaching the 


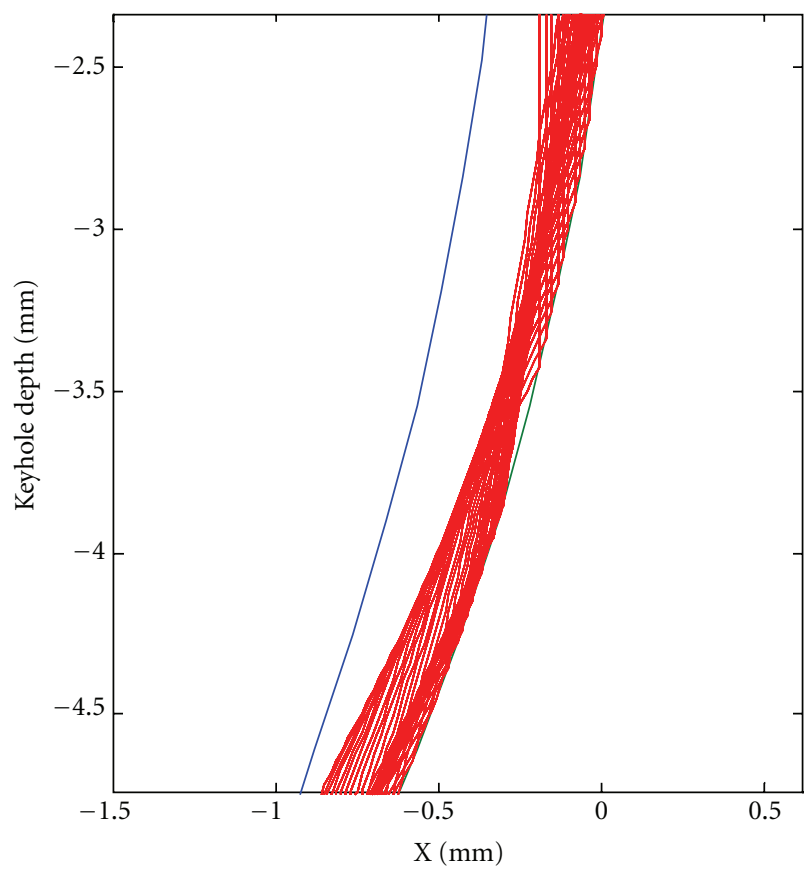

Figure 10: Diagram of some part of Figure 8 from about depth $2.4 \mathrm{~mm}$ to $4.8 \mathrm{~mm}$.

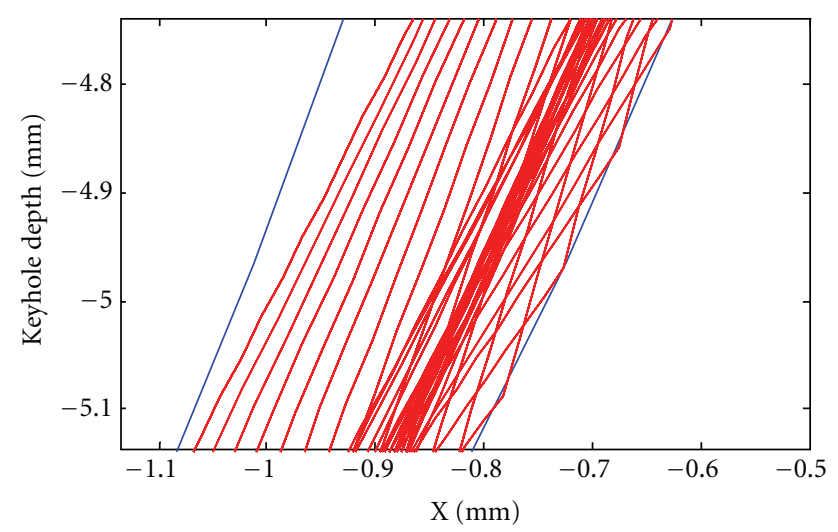

FIGURE 11: Diagram of some part of Figure 8 from depth $4.8 \mathrm{~mm}$ to $5.1 \mathrm{~mm}$.

highest value of $6.5 \times 10^{8} \mathrm{~W} / \mathrm{m}^{2}$ at the keyhole tail. In other words, due to more frequent multiple reflections of laser rays, the laser intensity absorbed at the keyhole bottom is higher than that on the upper part.

Comparing Figure 12 with the results of [13], it can be seen clearly that, with Gaussian optics theory, the keyhole wall can absorb extensive laser intensity even at the keyhole mouth. Maybe this is another answer to the question of what mechanism keeps the keyhole open in such regions without enough absorbed laser intensity in [13]. Because of Gaussian distribution of laser beam, the keyhole wall, even at keyhole mouth, can absorb laser power which is responsible for keeping the keyhole open.

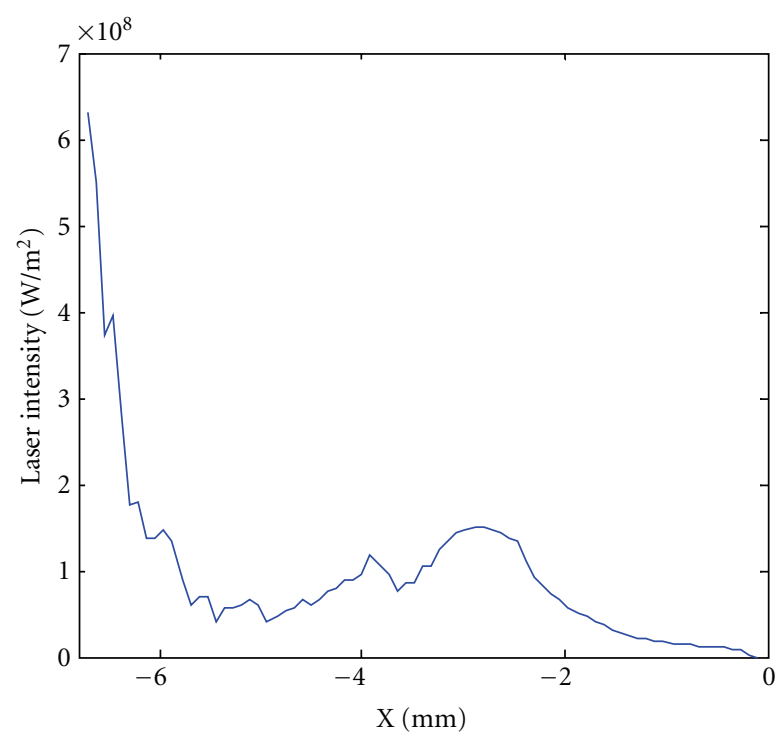

FIgURE 12: The average laser intensity absorbed along keyhole depth.

\section{Conclusions}

(1) The distribution of the absorbed laser intensity on keyhole profile is very uneven. On the upper side of the keyhole, far more laser intensity is absorbed by the front keyhole wall, while the rear keyhole wall can only absorb little weak laser intensity. However, at the bottom of the keyhole, due to multiple reflections, the front and rear keyhole wall absorbs almost the same level of laser intensity.

(2) Due to more frequent multiple reflections and the Fresnel absorption, the keyhole bottom can absorbed even more laser intensity than that on the upper of the keyhole. The higher the absorbed laser intensity, the stronger the vaporization and the bigger the recoil pressure, which is the main cause for keeping the keyhole open at the bottom.

(3) Under the Gaussian optics theory, even some area near the keyhole mouth can absorb some laser intensity.

\section{Acknowledgments}

The authors of this paper would like to thank both the National Natural Science Foundation of China under the Grant number 50875079 and the Natural Science Foundation of Hunan Province of China under the Grant number 09JJ3088 for giving them financial support to pursue this research.

\section{References}

[1] J. Dowden, P. Kapadia, and N. Postacioglu, "Analysis of the laser-plasma interaction in laser keyhole welding," Journal of Physics D, vol. 22, no. 6, pp. 741-749, 1989. 
[2] M. Beck, P. Berger, F. Dausinger, and H. Hugel, "Aspects of keyhole/melt interaction in high-speed laser welding," in 8th International Symposium on Gas Flow and Chemical Lasers, vol. 1397 of Proceedings of SPIE, pp. 769-774, 1991.

[3] M. Kern, Simulation der absorption polarisierter beim LaserTiefschweißen, Studienarbeit, Universität Stuttgart, Deutchland, 1992.

[4] M. Mueller, Simulation der reflektierten Laserstrahlung zur uslegung einer Prozeßkontrolle fuer das Laserschweißen Diplomarbeit, University at Stuttgart, Deutchland, 1996.

[5] A. Kaplan, "A model of deep penetration laser welding based on calculation of the keyhole profile," Journal of Physics D, vol. 27, pp. 1805-1814, 1994.

[6] P. Solana and G. Negro, "A study of the effect of multiple reflections on the shape of the keyhole in the laser processing of materials," Journal of Physics D, vol. 30, no. 23, pp. 3216-3222, 1997.

[7] R. Fabbro and K. Chouf, "Keyhole description in deep-penetration laser welding," in High-Power Lasers in Manufacturing, vol. 3888 of Proceedings of SPIE, pp. 104-112, 2000.

[8] V. V. Semak, J. A. Hopkins, M. H. McCay, and T. D. McCay, "Melt pool dynamics during laser welding," Journal of Physics D, vol. 28, no. 12, pp. 2443-2450, 1995.

[9] S. Katayama, N. Seto, J. D. Kim, and A. Matsunawa, "Formation mechanism and reduction method of porosity in laser welding of stainless steel," in Proceedings of the Laser Materials Processing Conference (ICALEO '97), Section G, pp. 83-92, San Diego, Calif, USA, November 1997.

[10] S. Fujinaga, H. Takenaka, T. Narikiyo, S. Katayama, and A. Matsunawa, "Direct observation of keyhole behaviour during pulse modulated high-power Nd:YAG laser irradiation," Journal of Physics D, vol. 33, no. 5, pp. 492-497, 2000.

[11] X. Jin, L. Li, and Y. Zhang, "A study on fresnel absorption and reflections in the keyhole in deep penetration laser welding," Journal of Physics D, vol. 35, no. 18, pp. 2304-2310, 2002.

[12] Y. Arata, H. Maruo, I. Miyamoto, and S. Takeuchi, "Dynamic behavior of laser welding and cutting," in Proceedings of the 7th International Conference on Electron and Ion Beam Science and Technology, pp. 111-128, San Francisco, Calif, USA, 1976.

[13] X. Jin, P. Berger, and T. Graf, "Multiple reflections and Fresnel absorption in an actual 3D keyhole during deep penetration laser welding," Journal of Physics D, vol. 39, no. 21, pp. 47034712, 2006.

[14] X. Jin, "A three-dimensional model of multiple reflections for high-speed deep penetration laser welding based on an actual keyhole," Optics and Lasers in Engineering, vol. 46, no. 1, pp. 83-93, 2008.

[15] C. Yuanyong, J. S. L. Xiangzhong, and Z. Licheng, "Fresnel absorption and inverse bremsstrahlung absorption in an actual $3 \mathrm{D}$ keyhole during deep penetration $\mathrm{CO}_{2}$ laser welding," Optics \& Laser Technology, vol. 44, pp. 1426-1436, 2012.

[16] H. Guo, Y. Zhang, and J. Zhang, "The experimental study of the reflection characteristics of Gaussian beam," Journal of Yanan University, vol. 14, no. 2, pp. 33-41, 1995. 

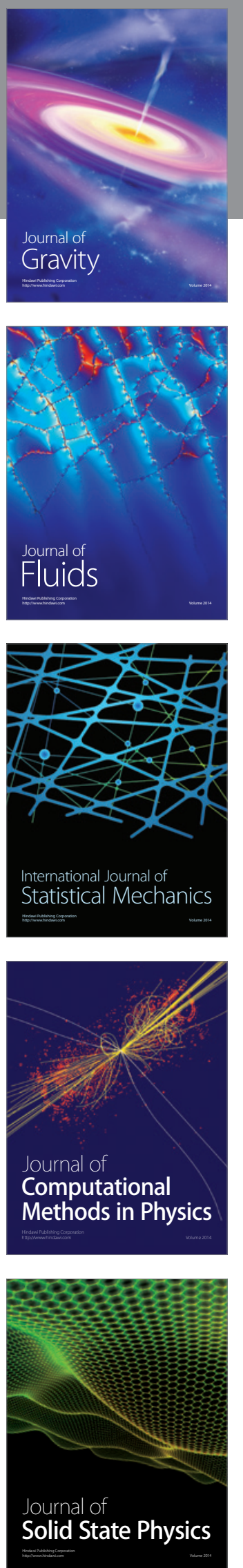

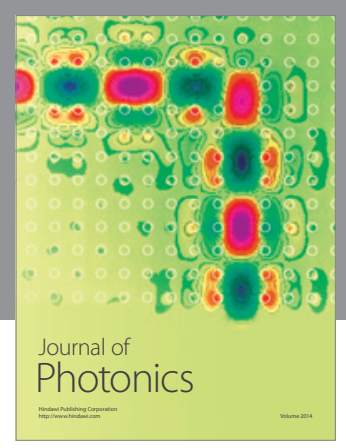

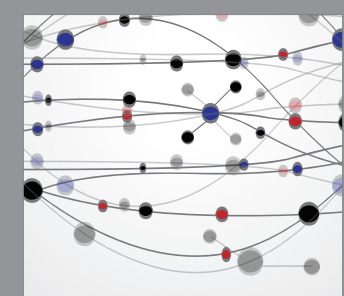

The Scientific World Journal
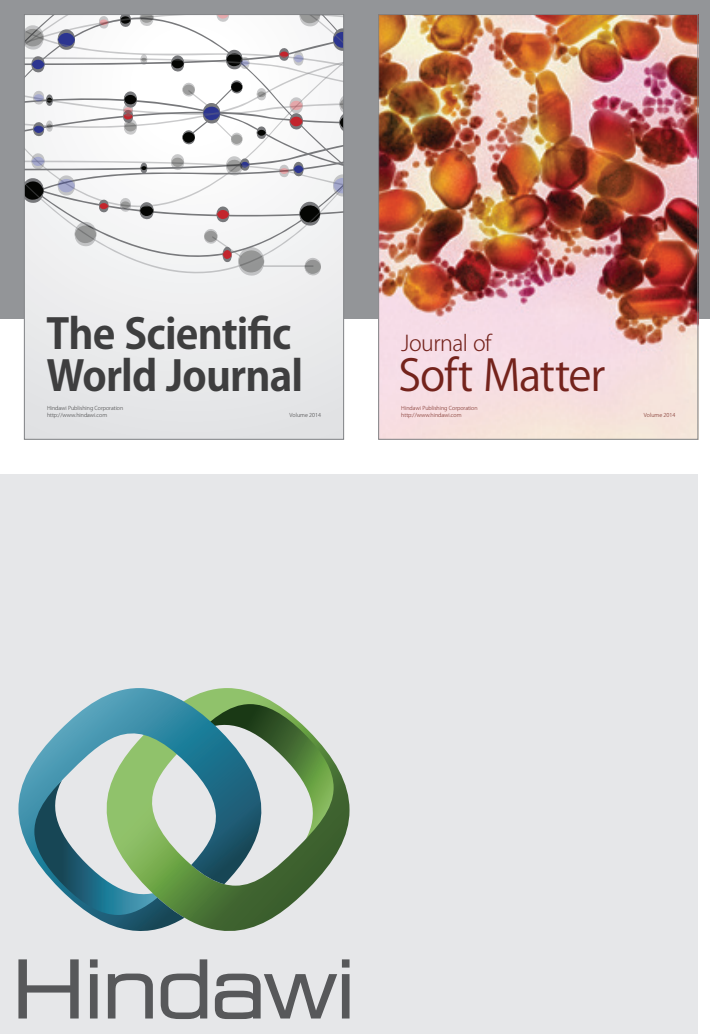

Submit your manuscripts at

http://www.hindawi.com
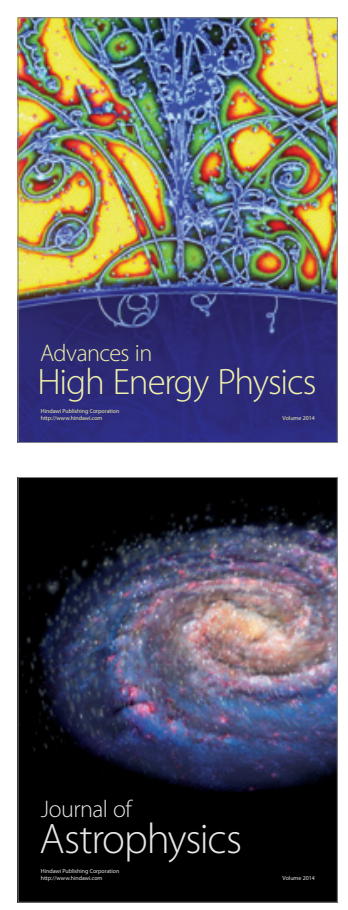
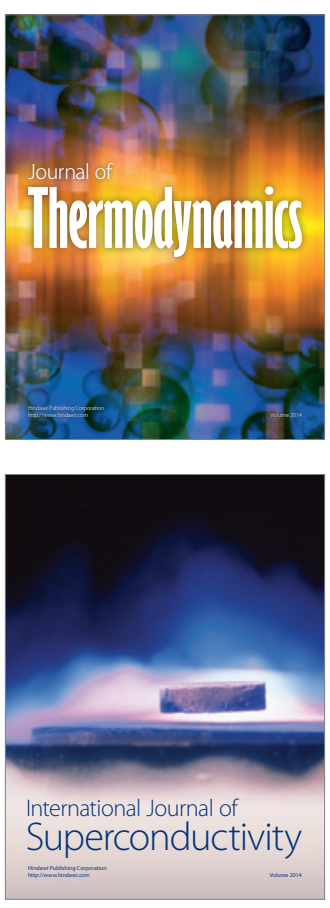
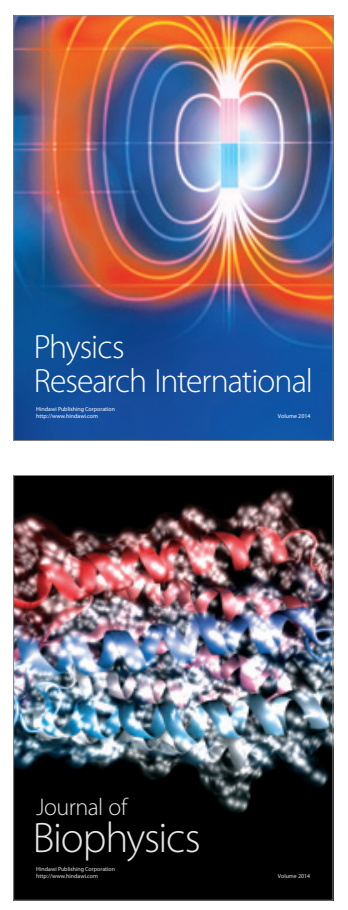
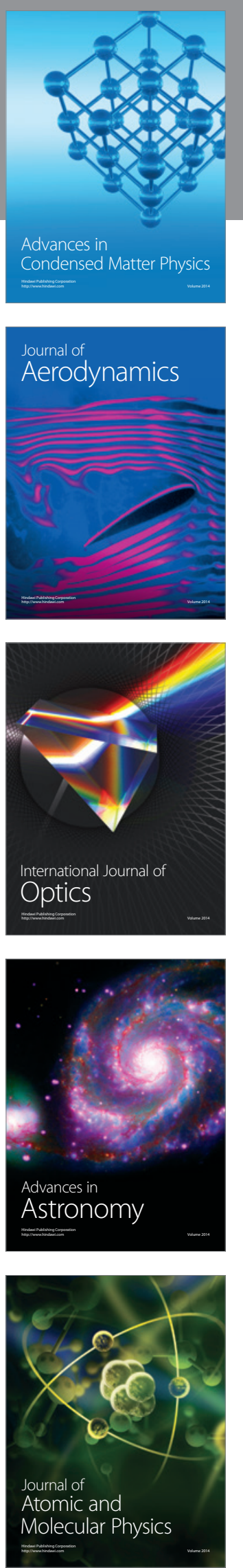